\title{
Diversity-Multiplexing Tradeoff-Optimal Code Constructions for Symmetric MIMO Multiple Access Channels
}

\author{
Hsiao-feng (Francis) Lu \\ Department of Communications Engineering \\ National Chiao Tung University \\ Hsinchu, Taiwan \\ E-mail: francis@cc.nctu.edu.tw
}

\author{
Camilla Hollanti \\ Department of Mathematics \\ University of Turku \\ FI-20014, Finland \\ E-mail: cajoho@utu.fi
}

\begin{abstract}
An explicit, systematic code construction for the symmetric MIMO (multi-input multi-output) multiple access (MAC) channel with any number of users and any numbers of transmit and receive antennas is presented in this paper. The users are assumed to transmit at the same level of multiplexing gain. This newly constructed code is proved to achieve the optimal MIMO-MAC diversity-multiplexing tradeoff.
\end{abstract}

\section{INTRODUCTION}

During the past five years extensive research has been carried out on point-to-point space-time (ST) lattice codes based on cyclic division algebras (CDAs) [1]-[4]. At its best, this research has resulted in codes having performance very close to outage bound for practical numbers of antennas and at moderate SNR regime. At high SNR regime, the CDA-based codes are shown to be optimal in terms of the diversity-multiplexing tradeoff (DMT) proposed by Zheng and Tse [5]. In the point-to-point scenario, let $n_{t}$ and $n_{r}$ be respectively the numbers of transmit and receive antennas at the transmitter and receiver ends. At high SNR regime, let $r, 0 \leq r \leq \min \left\{n_{t}, n_{r}\right\}$, denote the multiplexing gain which means the actual transmission rate equals $R=r \log _{2}$ SNR bits per channel use. It was shown [2] that at multiplexing gain $r$, the CDA-based codes are capable of achieving the following optimal error probability

$$
P_{\text {cwe }}(\mathrm{SNR}) \doteq \mathrm{SNR}^{-d_{n_{t}, n_{r}}^{*}(r)}
$$

where $\doteq$ denotes the exponential equality defined in [5]. The exponent $d_{n_{t}, n_{r}}^{*}(r)$ is a piecewise linear function connecting the points $\left(r,\left(n_{t}-r\right)\left(n_{r}-r\right)\right)$ for $r=0,1, \cdots, \min \left\{n_{t}, n_{r}\right\}$. In fact, $d_{n_{t}, n_{r}}^{*}(r)$ is the best possible diversity gain that can be achieved by any point-to-point space-time codes under Rayleigh block fading channel whenever the channel coherence time is $\geq n_{t}$ channel uses [2]. The function $d_{n_{t}, n_{r}}^{*}(r)$ is therefore commonly known as the DMT.

Motivated by the promising outcome in the point-to-point scenario, the aim of this paper is to investigate the code construction for the multiple-access (MAC) scenario, or equivalently, the uplink transmission of multiuser (MU) MIMO communication. We will concentrate on the uplink transmission from multiple users to a single base station (or access point). Both mobile users and base station may be equipped with multiple antennas.

Consider a MIMO MAC channel with $K$ mobile users, each user equipped with $n_{t}$ transmit antennas, and $n_{r}$ antennas available at base station. Assuming each user communicating independently to the base station, the DMT of such MIMOMAC channel was first studied by Tse et al. in [6]. To distinguish from the DMT of point-to-point scenario, we will call it MAC-DMT henceforth. The problem of constructing MAC-DMT optimal codes is to construct a coding scheme to "independently" encode and transmit each mobile user's information across the MIMO channel such that at the receiver end, i.e. base station, the joint decoding of all users' signals reaches the best possible error performance, which is characterized by the MAC-DMT. To simplify the problem, here we consider only the case when all users transmit at the same level of multiplexing gain $r$. This was termed the symmetric MIMO-MAC channel in [6]. Furthermore, Tse et al. [6] proved that when each user's information is independently coded, the maximal possible diversity gain is given by

$d_{n_{t}, n_{r}, K}^{*}(r)=\left\{\begin{array}{l}d_{n_{t}, n_{r}}^{*}(r), \quad \text { if } r \in\left[0, \min \left\{n_{t}, \frac{n_{r}}{K+1}\right\}\right] \\ d_{K n_{t}, n_{r}}^{*}(K r), \\ \quad \text { if } r \in\left[\min \left\{n_{t}, \frac{n_{r}}{K+1}\right\}, \min \left\{n_{t}, \frac{n_{r}}{K}\right\}\right] .\end{array}\right.$

Notice that $r$ is bounded between

$$
0 \leq r \leq \min \left\{n_{t}, \frac{n_{r}}{K}\right\}
$$

Compared with the point-to-point scenario, the decrease by a factor of $K$ on the right-hand-side of (3) is because of $K$ users in the MAC channel. (2) also reveals that when the level of multiplexing gain is low, i.e. $r \in$ $\left[0, \min \left\{n_{t}, \frac{n_{r}}{K+1}\right\}\right]$, the decoding of each user's signal is able to achieve single-user performance, that is, it is as if there were no other users present in MAC channel. On the other hand, when the level of multiplexing gain is high, 
meaning $r \in\left[\min \left\{n_{t}, \frac{n_{r}}{K+1}\right\}, \min \left\{n_{t}, \frac{n_{r}}{K}\right\}\right]$, the whole MIMO-MAC system will be in the antenna pooling region [6], and the single-user performance can never be maintained. As a consequence, a much lower diversity gain $d_{K n_{t}, n_{r}}^{*}(K r)$ will dominate the error performance in this regime. Thus, in this paper we will focus on constructing MIMO-MAC codes whose diversity gain performance achieves (2) at high SNR regime.

Some works have been done in this area when there are $K=2$ users present in the MIMO MAC channel. Based on pairwise error probability analysis, [7] extended the design criteria of point-to-point ST codes to the multiuser case, and introduced an explicit $(2 \times 2)$ two-user MIMO construction using independent Alamouti blocks. Yet, the codes in [7] do not achieve the optimal MAC-DMT. Nam et al. [8] proposed the first MAC-DMT achieving transmission scheme based on a class of structured multiple access random lattice ST codes. Some explicit, algebraic code constructions for $n_{t}>1$ and $K=2$ were introduced in [9] and [10]. In [9] the authors proposed a design criteria based on a truncated union bound approximation, and with the aid of these criteria they managed to outperform in error performance the other known two-user codes for the $(2 \times 2)$ MAC [7], [11]. The authors of [10] proposed another code construction for the $(2 \times 2)$ two-user MAC code by introducing a rotation matrix $\Gamma$ to the original point-to-point CDA-based ST code such that the overall code matrix is nonsingular. However, this $\Gamma$ matrix causes the resulting code to be ill-conditioned, i.e. having vanishing determinant, at high SNR regime.

In this paper, we will present a new code construction for the symmetric MIMO-MAC channel for any numbers of transmit and receive antennas and for any number of users. In particular, we will prove that this newly proposed construction is MAC-DMT optimal, i.e. it achieves the optimal diversity gain given in (2) whenever all users transmit at the same level of multiplexing gain $r$. This paper is organized as follows. The proposed construction will be given in Section II, and several nice properties possessed by the code will be presented. Due to limited space, a brief outline of the proof of the MAC-DMT optimality will be given in Section III.

\section{MAC-DMT Optimal CODE CONSTRUCTION FOR MIMO-MAC SYSTEMS}

Consider a MIMO-MAC channel consisting of $K$ users. Each user is equipped with $n_{t}$ transmit antennas, and communicates independently to the base station that has $n_{r}$ receive antennas. We further assume that all users transmit at the same level of multiplexing gain $r$. Given the setting above, the proposed construction is the following. Firstly, given the number of users $K$, let $K_{o}$ be the smallest odd integer such that $K_{o} \geq K$, i.e.

$$
K_{o}=\left\{\begin{array}{cl}
K+1, & \text { if } K \text { even, } \\
K, & \text { if } K \text { odd. }
\end{array}\right.
$$

The code construction then calls for the following number fields. Let $\mathbb{K}_{o}=\mathbb{F}\left(\eta_{o}\right)$ be the number field that is a cyclic Ga-

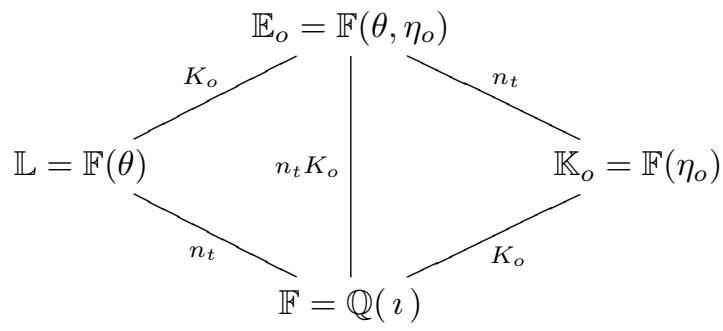

Fig. 1. Field extensions required by the proposed code constructions.

lois extension of $\mathbb{F}=\mathbb{Q}(\imath)$ with degree $K_{o}$, and that satisfies $\mathbb{K}_{o} \cap \mathbb{L}=\mathbb{F}$, where $\mathbb{L}=\mathbb{F}(\theta)$ is a cyclic Galois extension of $\mathbb{F}$ with degree $n_{t}$. Let $\sigma$ and $\tau_{o}$ be generators of $\operatorname{Gal}(\mathbb{L} / \mathbb{F})$ and $\operatorname{Gal}\left(\mathbb{K}_{o} / \mathbb{F}\right)$ with degrees $n_{t}$ and $K_{o}$, respectively. Let $\mathbb{E}_{o}=\mathbb{K}_{o} \mathbb{L}=\mathbb{F}\left(\eta_{o}, \theta\right)$. See Fig. 1 for the relation between the required number fields. Let $\mathfrak{D}_{o}=\left(\mathbb{E}_{o} / \mathbb{K}_{o}, \sigma, \zeta=\frac{\gamma}{\gamma^{*}}\right)$ be a cyclic division algebra,

$$
\mathfrak{D}_{o}=\mathbb{E}_{o} \oplus z \mathbb{E}_{o} \oplus \cdots \oplus z^{n_{t}-1} \mathbb{E}_{o},
$$

with

$$
x z=z \sigma(x)
$$

for $x \in \mathbb{E}_{o}$, where $z$ is an indeterminate satisfying $z^{n_{t}}=$ $\zeta \in \mathbb{F}$, and $0 \neq \gamma \in \mathcal{O}_{\mathbb{F}}$ is some suitable nonnorm element. By $\gamma^{*}$ we mean the complex conjugate of $\gamma$ and $\mathcal{O}_{\mathbb{F}}$ is the ring of algebraic integers in field $\mathbb{F}$. Notice that $\|\zeta\|=1$ and that $\zeta$ is unimodular. It has been shown [3] that with such unimodular $\zeta, \mathfrak{D}_{O}$ is always a cyclic division algebra. Let $\psi_{o}: \mathfrak{D}_{o} \rightarrow M_{n_{t}}\left(\mathbb{E}_{o}\right)$ be the left-regular map that maps elements in $\mathfrak{D}_{o}$ into $\left(n_{t} \times n_{t}\right)$ matrices with entries in $\mathbb{E}_{o}$.

Given multiplexing gain $r$, let $\mathcal{A}(\mathrm{SNR})$ be the base alphabet defined as below

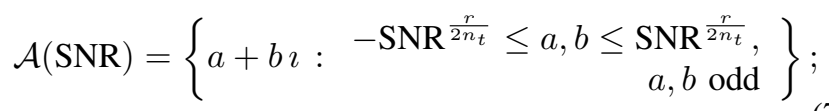

then the corresponding information set is given by

$$
\mathfrak{A}_{o}(\mathrm{SNR})=\left\{\sum_{i=0}^{n_{t}-1} z^{i} \sum_{k=0}^{K_{o} n_{t}-1} x_{i, k} e_{k}: x_{i, k} \in \mathcal{A}(\mathrm{SNR})\right\},
$$

where $\left\{e_{0}, \cdots, e_{K_{o} n_{t}-1}\right\}$ is an integral basis of $\mathbb{E}_{o} / \mathbb{F}$.

Having set the above, the information encoding of each user's data stream will proceed as follows. Given the multiplexing gain $r$, the $i$ th user first divides its binary data steams into blocks of $r K_{o} n_{t} \log _{2}$ SNR bits. Then by using the integral basis $\left\{e_{0}, \cdots, e_{K_{o} n_{t}-1}\right\}$ and the sets $\mathcal{A}(\mathrm{SNR})$ and $\mathfrak{A}_{o}(\mathrm{SNR})$ defined above, each block of binary bits can be mapped to some symbol $x_{i} \in \mathfrak{A}_{o}(\mathrm{SNR})$ in an one-one fashion. Notice that each user encodes his/her information independently.

Given the information symbol $x_{i} \in \mathfrak{A}_{o}(\mathrm{SNR})$ of the $i$ th user, the corresponding $\left(n_{t} \times K_{o} n_{t}\right)$ signal matrix $S_{i}$ that is actually sent out through the user $i$ 's transmit antenna array is given by

$$
S_{i}=\kappa\left[\begin{array}{llll}
X_{i} & \tau_{o}\left(X_{i}\right) & \cdots & \tau_{o}^{K_{o}-1}\left(X_{i}\right)
\end{array}\right],
$$


where $X_{i}=\psi_{o}\left(x_{i}\right)$ and where

$$
\kappa^{2} \doteq \mathrm{SNR}^{1-\frac{r}{n_{t}}}
$$

is the normalizing constant such that the average signalto-noise power ratio is SNR. The transmission takes $n_{t} K_{o}$ channel uses to complete.

The overall space-time code for $K$ users is given by

$$
\mathcal{S}=\left\{S=\kappa\left[\begin{array}{ccc}
X_{0} & \cdots & \tau_{o}^{K_{o}-1}\left(X_{0}\right) \\
\vdots & \ddots & \vdots \\
X_{K-1} & \cdots & \tau_{o}^{K_{o}-1}\left(X_{K-1}\right)
\end{array}\right]:\right.
$$

For the purpose of code performance analysis that comes later we set $\mathcal{C}=\frac{1}{\kappa} \mathcal{S}$, i.e.

$$
\mathcal{C}=\left\{C=\left[\begin{array}{ccc}
X_{0} & \cdots & \tau_{o}^{K_{o}-1}\left(X_{0}\right) \\
\vdots & \ddots & \vdots \\
X_{K-1} & \cdots & \tau_{o}^{K_{o}-1}\left(X_{K-1}\right)
\end{array}\right]:\right.
$$

Let $H_{i}$ be the $\left(n_{r} \times n_{t}\right)$ channel matrix of the $i$ th user, and here we assume $H_{i}$ is fixed for a block of $n_{t} K_{o}$ channel uses. Entries of $H_{i}$ are modeled as i.i.d. complex Gaussian random variables $\mathbb{C} \mathcal{N}(0,1)$ to represent the Rayleigh block fading phenomenon. Thus given $S \in \mathcal{S}$, the received signal matrix at the receiver end is

$$
\left[\begin{array}{lll}
Y_{0} & \cdots & Y_{K_{o}-1}
\end{array}\right]=\left[\begin{array}{lll}
H_{0} & \cdots & H_{K-1}
\end{array}\right] S+W,
$$

where $W$ is the noise matrix whose entries are i.i.d. $\mathbb{C} \mathcal{N}(0,1)$ random variables, and $Y_{j}$ is the $j$ th block received signal matrix given by

$$
Y_{j}=\sum_{k=0}^{K-1} H_{k} \kappa \tau_{o}^{j}\left(X_{k}\right)+W, \quad j=0,1, \cdots, K_{o}-1
$$

\section{A. Properties of the Proposed Construction}

To simplify the analysis of the code performance, below we define the extended version of codes $\mathcal{S}$ and $\mathcal{C}$.

$$
\overline{\mathcal{C}}_{o}=\left\{\bar{C}_{o}=\left[\begin{array}{ccc}
X_{0} & \cdots & \tau_{o}^{K_{o}-1}\left(X_{0}\right) \\
\vdots & \ddots & \vdots \\
X_{K_{o}-1} & \cdots & \tau_{o}^{K_{o}-1}\left(X_{K_{o}-1}\right)
\end{array}\right]:\right.
$$

$\overline{\mathcal{S}}_{o}=\left\{\bar{S}_{o}=\kappa \bar{C}_{o}: \bar{C}_{o} \in \overline{\mathcal{C}}_{o}\right\}$

It is clear that both $\overline{\mathcal{S}}_{o}$ and $\overline{\mathcal{C}}_{o}$ are sets of square matrices having size $\left(K_{o} n_{t} \times K_{o} n_{t}\right)$. In particular, we have $\overline{\mathcal{S}}_{o}=\mathcal{S}$ and $\overline{\mathcal{C}}_{o}=\mathcal{C}$ if $K$ odd. Furthermore, given the overall signal matrix $S \in \mathcal{S}$, let $\bar{S}_{o} \in \overline{\mathcal{S}}_{o}$ be any signal matrix whose first
$K n_{t}$ rows equals $S$. Then we can rewrite the received signal matrix of (12) as

$$
\left[\begin{array}{lll}
Y_{0} & \cdots & Y_{K_{o}-1}
\end{array}\right]=\left[\begin{array}{lll}
H_{0} & \cdots & H_{K_{o}-1}
\end{array}\right] \bar{S}_{o}+W
$$

where

$$
H_{K_{o}-1}=\left\{\begin{array}{cl}
H_{K-1}, & \text { if } K \text { odd, } \\
\mathbf{0}, & \text { if } K \text { even. }
\end{array}\right.
$$

As (12) and (16) are equivalent, below we will work only with the extended codes $\overline{\mathcal{S}}_{o}$ and $\overline{\mathcal{C}}_{o}$, rather than $\mathcal{S}$ and $\mathcal{C}$.

Property 1: For any $\bar{C}_{o} \in \overline{\mathcal{C}}_{o}$

$$
\left[\left(\gamma^{*}\right)^{K_{o}\left(n_{t}-1\right)} \operatorname{det}\left(\bar{C}_{o}\right)\right] \in \mathbb{Z}[\imath]
$$

Proof (Sketch): It can be shown that $\operatorname{det}\left(\bar{C}_{o}\right)$ is fixed under the automorphisms, hence $\operatorname{det}\left(\bar{C}_{o}\right) \in \mathbb{F}=\mathbb{Q}(\imath)$. Next, for each component matrix $\tau_{o}^{j}\left(X_{i}\right)$ of $\bar{C}_{o}$, it can be seen that the product matrix $\operatorname{diag}\left(1, \gamma^{*}, \cdots, \gamma^{*}\right) \tau_{o}^{j}\left(X_{i}\right)$ has entries in $\mathcal{O}_{\mathbb{E}_{o}}$ for every $j=0,1, \cdots, K_{o}-1$, hence $\left[\left(\gamma^{*}\right)^{K_{o}\left(n_{t}-1\right)} \operatorname{det}\left(\bar{C}_{o}\right)\right] \in \mathcal{O}_{\mathbb{E}_{o}}$. Finally, we have $\mathcal{O}_{\mathbb{E}_{o}} \cap$ $\mathbb{Q}(\imath)=\mathbb{Z}[\imath]$ and this completes the proof.

Property 2: Let

$$
\mathfrak{C}=\left[\begin{array}{c}
\underline{x}_{0}^{t} \\
\vdots \\
\underline{x}_{K_{o}-1}^{t}
\end{array}\right]=\left[\begin{array}{ccc}
x_{0} & \cdots & \tau_{o}^{K_{o}-1}\left(x_{0}\right) \\
\vdots & \ddots & \vdots \\
x_{K_{o}-1} & \cdots & \tau_{o}^{K_{o}-1}\left(x_{K_{o}-1}\right)
\end{array}\right]
$$

and

$$
\bar{C}_{o}=\left[\begin{array}{ccc}
X_{0} & \cdots & \tau_{o}^{K_{o}-1}\left(X_{0}\right) \\
\vdots & \ddots & \vdots \\
X_{K_{o}-1} & \cdots & \tau_{o}^{K_{o}-1}\left(X_{K_{o}-1}\right)
\end{array}\right]
$$

with $X_{i}=\psi_{o}\left(x_{i}\right), x_{i} \in \mathfrak{A}_{o}(\mathrm{SNR})$. Let $m$ be the maximal number such that the set $\left\{\underline{x}_{i_{1}}^{t}, \cdots, \underline{x}_{i_{m}}^{t}\right\}$ is linearly independent as a left $\mathfrak{D}_{o}$ module, where $\left\{i_{1}, \cdots, i_{m}\right\} \subseteq$ $\left\{0,1, \cdots, K_{o}-1\right\}$; then

$$
\operatorname{rank}\left(\bar{C}_{o}\right)=m n_{t}
$$

where the rank is measured in the complex number field $\mathbb{C}$. Proof (Sketch): Notice that although $\mathfrak{D}_{o}$ is noncommutative, it is still possible to apply Gauss-Jordan elimination on $\mathfrak{C}$. Secondly, applying elementary row reduction on $\mathfrak{C}$ is equivalent to applying elementary block reduction over $\left(n_{t} \times n_{t}\right)$ blocks of $\bar{C}_{o}$.

The above property shows that the overall code matrix $\bar{C}_{o} \in \overline{\mathcal{C}}_{o}$ does not always have full rank $K_{o} n_{t}$, and the rank of $\bar{C}_{o}$ is always a multiple of $n_{t}$. This is not too much of a surprise as it is straightforward to see that in (14) if some of the matrices $X_{i}$ are identical, then the overall code matrix $\bar{C}_{o}$ cannot be nonsingular. However, compared with the constructions proposed in [9], [10], the code matrix $\bar{C}_{o}$ defined in (14) can be singular even when the component matrices $X_{i}$ are all distinct as shown in the proof of Property 2. Nevertheless, we will prove in Section III that in order to achieve the optimal MAC-DMT performance at high SNR regime, it 
is unnecessary for the code construction such that the code matrix $\bar{C}_{o}$ is nonsingular whenever all component matrices $X_{i}$ are distinct. Before rigorously proving this claim, a heuristic way to see this is the following. Since each user communicates independently to the base station, for any overall MIMO-MAC code $\mathcal{C}$ it is impossible for all the code matrices $C \in \mathcal{C}$ to be nonsingular. Also, from the pairwise error probability of view, for any $C \neq C^{\prime} \in \mathcal{C}, C-C^{\prime}$ can be singular at least when some of the users' data are the same, and the corresponding rank is at best a multiple of $n_{t}$. Therefore, intuitively speaking, perhaps it does not hurt to make things a bit worse in the sense that the difference matrix $C-C^{\prime}$ can be singular in other cases. The reason for this is that in the MAC-DMT (2) the dominant error events are the ones with probabilities $\mathrm{SNR}^{-d_{n_{t}, n_{r}}^{*}(r)}$ and $\mathrm{SNR}^{-d_{K_{o} n_{t}, n_{r}}^{*}\left(K_{o} r\right)}$, not the ones in between, i.e. not those of error probability SNR ${ }^{-d_{m n_{t}, n_{r}}^{*}(m r)}$ for $m=2,3, \cdots, K_{o}-1$. This means we should focus on the error events that are dominant, and we only have to make sure that the nondominant events are not too much worse in error performance. In other words, we do not need the code to behave at the same error performance as that of the Gaussian random code at every level of $m$.

On the other hand, even though we do not need the whole code $\overline{\mathcal{C}}_{o}$ to satisfy the nonvanishing determinant (NVD) property as in the point-to-point scenario, an alternative NVDlike property is preferred and is given as below.

Property 3: Let $\mathfrak{C}$ be defined as in (19) and assume that $\left\{\underline{x}_{i_{1}}^{t}, \cdots, \underline{x}_{i_{m}}^{t}\right\}$ is the maximal subset of rows of $\mathfrak{C}$ that are linearly independent as a left $\mathfrak{D}_{o}$ module. Define

$$
\mathfrak{C}_{s}=\left[\begin{array}{c}
\underline{x}_{i_{1}}^{t} \\
\vdots \\
\underline{x}_{i_{m}}^{t}
\end{array}\right] \quad \text { and } \bar{C}_{s}=\Psi_{o}\left(\mathfrak{C}_{s}\right)
$$

i.e. $\bar{C}_{s}$ is the submatrix of $\bar{C}_{o}$ consisting of $m n_{t}$ rows, where $\Psi_{o}$ is the natural extension of $\psi_{o}$. Then

$$
1<\left[\|\gamma\|^{2 m n_{t}} \cdot \operatorname{det}\left(\bar{C}_{s} \bar{C}_{s}^{\dagger}\right)\right] \in \mathbb{Z} .
$$

Proof (Sketch): The key is to show that $\operatorname{det}\left(\bar{C}_{s} \bar{C}_{s}^{\dagger}\right)$ is fixed under the automorphisms $\tau_{o}$ and $\sigma$. Due to limited space, we only give the sketch of proving the part $\sigma$ here. Firstly, with some omitted steps, it can be shown that

$$
\sigma\left(\bar{C}_{s}\right)=\left[\begin{array}{ccc}
Z^{-1} & & \\
& \ddots & \\
& & Z^{-1}
\end{array}\right] \bar{C}_{s}\left[\begin{array}{ccc}
Z & & \\
& \ddots & \\
& & Z
\end{array}\right],
$$

where $Z=\psi_{o}(z)$. It then can be shown that $Z Z^{\dagger}=I_{n_{t}}$ due to $\zeta \zeta^{*}=1$ by construction, and $Z^{-1}\left(Z^{-1}\right)^{\dagger}=I_{n_{t}}$. Thus, we see

$$
\begin{aligned}
& \sigma\left(\bar{C}_{s}\right)\left[\sigma\left(\bar{C}_{s}\right)\right]^{\dagger} \\
= & \operatorname{diag}\left(Z^{-1}, \cdots, Z^{-1}\right) \bar{C}_{s} \bar{C}_{s}^{\dagger} \operatorname{diag}\left(\left(Z^{-1}\right)^{\dagger}, \cdots,\left(Z^{-1}\right)^{\dagger}\right)
\end{aligned}
$$

and hence $\sigma\left[\operatorname{det}\left(\bar{C}_{s} \bar{C}_{s}^{\dagger}\right)\right]=\operatorname{det}\left(\bar{C}_{s} \bar{C}_{s}^{\dagger}\right)$.

With the three key properties satisfied by the proposed code construction $\overline{\mathcal{C}}_{o}$ of (14), we are able to show that the same code $\overline{\mathcal{S}}_{o}$ of (15) achieves the optimal MAC-DMT performance $d_{n_{t}, n_{r}, K}^{*}(r)$ for both $K$ even and odd.

\section{Proof Outline of MAC-DMT Optimality}

In this section we will give a sketch of the proof of the MAC-DMT optimality achieved by $\overline{\mathcal{C}}_{o}$. For brevity, we will focus only on the case of odd $K$.

In a nut-shell, given the overall channel matrix $H=$ $\left[H_{0} H_{1} \cdots H_{K_{o}-1}\right]$ the idea of the proof is to examine the minimum Euclidean distance of the noise-free received code $H \overline{\mathcal{S}}_{o}:=\left\{H \bar{S}_{o}: \bar{S}_{o} \in \overline{\mathcal{S}}_{o}\right\}$, that is, to determine

$$
d_{E, \min }(H):=\min _{\bar{S}_{o} \neq \bar{S}_{o}^{\prime} \in \overline{\mathcal{S}}_{o}}\left\|H \bar{S}_{o}-H \bar{S}_{o}^{\prime}\right\|_{F},
$$

where by $\|A\|_{F}$ we mean the Frobenius norm of matrix $A$. Having obtained $d_{E, \min }(H)$, the codeword error probability achieved by $\overline{\mathcal{S}}_{o}$ is upper bounded by

$$
\begin{aligned}
P_{\text {cwe }}(r \mid H) & \leq \operatorname{Pr}\left\{\|W\|_{F}^{2} \geq \frac{d_{E, \min }^{2}(H)}{4}\right\} \\
& =e^{-\frac{d_{E, \text { min }(H)}^{2}}{4}} \sum_{j=0}^{K_{o} n_{r} n_{t}-1} \frac{\left(d_{E, \min }^{2}(H)\right)^{j}}{j !},
\end{aligned}
$$

where $W$ is the $\left(n_{r} \times K_{o} n_{t}\right)$ noise matrix defined in (12). It should be noted that $d_{E, \min }(H)$ is a random variable that depends upon the overall channel matrix $H$. Setting

$$
d_{E, \min }^{2}(H):=\operatorname{SNR}^{\delta_{E, \min }(H)}
$$

we see

$$
\operatorname{Pr}\left\{\|W\|_{F}^{2} \geq \frac{d_{E, \min }^{2}(H)}{4}\right\} \doteq 0, \text { if } \delta_{E, \min }(H)>0 .
$$

As $P_{\text {cwe }}(r \mid H) \leq 1$ we can upper bound the codeword error probability by

$$
P_{\text {cwe }}(r) \leq \operatorname{Pr}\left\{\delta_{E, \min }(H) \leq 0\right\} .
$$

Thus the goal here is to show the following

$$
\operatorname{Pr}\left\{\delta_{E, \min }(H) \leq 0\right\} \dot{\leq} \mathrm{SNR}^{-d_{n_{t}, n_{r}, K}^{*}(r)}
$$

where the MAC-DMT $d_{n_{t}, n_{r}, K}^{*}(r)$ is defined in (2).

To this end, below we will proceed to determine the value of $d_{E, \min }(H)$ over all possible distinct pairs $\left(\bar{S}_{o}, \bar{S}_{o}^{\prime}\right)$. We distinguish two cases.

1) $x_{\ell} \neq x_{\ell}^{\prime}$ for $\ell \in\left\{i_{1}, \cdots, i_{m}\right\}$ and $x_{\ell}=x_{\ell}^{\prime}$ otherwise in (19). Further we assume $\operatorname{rank}\left(\bar{C}_{o}-\bar{C}_{o}^{\prime}\right)=m n_{t}$ : In other words, here we consider the case when out of $K_{o} x_{\ell}$ 's, $m$ of them are distinct and the $m$ rows $\left[\left(x_{\ell}-x_{\ell}^{\prime}\right) \cdots \tau_{o}^{K_{o}-1}\left(x_{\ell}-x_{\ell}^{\prime}\right)\right]$ formed by such $x_{\ell}$ 's are all linearly independent over $\mathfrak{D}_{o}$ as left $\mathfrak{D}_{o}$-modules.

In this case, let $\bar{C}_{s}$ and $\bar{C}_{s}^{\prime}$ be defined as in (21) and let

$$
H_{s}=\left[H_{i_{1}} \cdots H_{i_{m}}\right]
$$

be the equivalent $\left(n_{r} \times m n_{t}\right)$ channel matrix; then we have

$$
\left\|H \bar{S}_{o}-H \bar{S}_{o}^{\prime}\right\|_{F}^{2}=\left\|\kappa H_{s}\left(\bar{C}_{s}-\bar{C}_{s}^{\prime}\right)\right\|_{F}^{2} .
$$


By repeatedly using arithmetic-mean geometric-mean inequality, and using the facts that

$$
\operatorname{det}\left[\left(\bar{C}_{s}-\bar{C}_{s}^{\prime}\right)\left(\bar{C}_{s}-\bar{C}_{s}^{\prime}\right)^{\dagger}\right] \geq \frac{1}{\|\gamma\|^{2 m n_{t}}} \doteq 1
$$

from Property 3 and $\left\|\bar{C}_{s}-\bar{C}_{s}^{\prime}\right\|_{F}^{2} \dot{\leq} \mathrm{SNR}^{\frac{r}{n_{t}}}$ by construction, it can be shown that

$$
\left\|H \bar{S}_{o}-H \bar{S}_{o}^{\prime}\right\|_{F}^{2} \geq \mathrm{SNR}^{\max _{1 \leq k \leq Q_{m}} \delta_{1, k}^{(m)}\left(\underline{\alpha}_{1}^{(m)}\right)}
$$

where $Q_{m}:=\min \left\{m n_{t}, n_{r}\right\}, k=1, \cdots, Q_{m}$,

$$
\delta_{1, k}^{(m)}\left(\underline{\alpha}_{1}^{(m)}\right):=\frac{1}{k}\left[\sum_{i=Q_{m}-k+1}^{Q_{m}}\left(1-\alpha_{1, i}^{(m)}\right)\right]-\frac{r m}{k},
$$

and where $\mathrm{SNR}^{-\alpha_{1,1}^{(m)}} \leq \cdots \leq \mathrm{SNR}^{-\alpha_{1, Q_{m}}^{(m)}}$ are the ordered nonzero eigenvalues of $H_{s} H_{s}^{\dagger}$.

2) The second case is when $\operatorname{rank}\left(\bar{C}_{o}-\bar{C}_{o}^{\prime}\right)=n n_{t}, n<m$, and without loss of generality we can assume

$$
\left\{\left[\left(x_{\ell}-x_{\ell}^{\prime}\right) \cdots \tau_{o}^{K_{o}-1}\left(x_{\ell}-x_{\ell}^{\prime}\right)\right]: \ell=i_{1}, \cdots, i_{n}\right\}
$$

are the maximal subset of left- $\mathfrak{D}_{o}$ linearly independent rows while $x_{\ell} \neq x_{\ell}^{\prime}$ for $\ell \in\left\{i_{1}, \cdots, i_{m}\right\}$ and $x_{\ell}=x_{\ell}^{\prime}$ otherwise.

Set $\Delta X_{\ell}=\psi_{o}\left(x_{\ell}-x_{\ell}^{\prime}\right)$; Property 2 then implies that

$$
\bar{C}_{s}-\bar{C}_{s}^{\prime}=\left[\begin{array}{ccc}
I_{n_{t}} & & \\
& \ddots & \\
& & I_{n_{t}} \\
P_{i_{n+1}, 1} & \cdots & P_{i_{n+1}, n} \\
\vdots & \vdots & \vdots \\
P_{i_{m}, 1} & \cdots & P_{i_{m}, n}
\end{array}\right] \boldsymbol{\Delta} \mathbf{X}
$$

where

$$
\Delta \mathbf{X}:=\left[\begin{array}{ccc}
\Delta X_{i_{1}} & \cdots & \tau_{o}^{K_{o}-1}\left(\Delta X_{i_{1}}\right) \\
\vdots & \ddots & \vdots \\
\Delta X_{i_{n}} & \cdots & \tau_{o}^{K_{o}-1}\left(\Delta X_{i_{n}}\right)
\end{array}\right]
$$

Given the channel matrix $H_{s}=\left[H_{i_{1}} \cdots H_{i_{m}}\right]$, we define the equivalent channel matrix $\mathbf{H}_{e q}=\left[\tilde{H}_{1} \cdots \tilde{H}_{n}\right]$ with

$$
\tilde{H}_{\ell}:=H_{i_{\ell}}+\sum_{k=n+1}^{m} H_{i_{k}} P_{i_{k}, \ell}
$$

for $\ell=1, \cdots, n$. Then it follows that

$$
\left\|H \bar{S}_{o}-H \bar{S}_{o}^{\prime}\right\|_{F}^{2}=\left\|\kappa \mathbf{H}_{e q} \mathbf{\Delta X}\right\|_{F}^{2} .
$$

Arguing similarly as the first case shows that

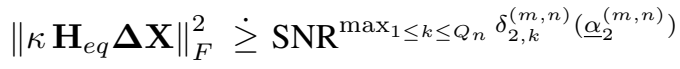

where

$$
\delta_{2, k}^{(m, n)}\left(\underline{\alpha}_{2}^{(m, n)}\right):=\frac{1}{k}\left[\sum_{i=Q_{n}-k+1}^{Q_{n}}\left(1-\alpha_{2, i}^{(m, n)}\right)\right]-\frac{r n}{k},
$$

$Q_{n}=\min \left\{n n_{t}, n_{r}\right\}, k=1, \cdots, Q_{n}$, and $\mathrm{SNR}^{-\alpha_{2, i}^{(m, n)}}$, $i=1, \cdots, Q_{n}$ are the ordered nonzero eigenvalues of $\mathbf{H}_{e q} \mathbf{H}_{e q}^{\dagger}$. It should be noted that $\delta_{2, k}^{(m, n)}\left(\underline{\alpha}_{2}^{(m, n)}\right)$ is exactly the same as $\delta_{1, k}^{(m)}\left(\underline{\alpha}_{1}^{(m)}\right)$ except that $\underline{\alpha}_{1}^{(m)}$ and $\underline{\alpha}_{2}^{(m, n)}$ are of different statistical distribution.

It is clear from the definition of $d_{E, \min }(H)$ that

$$
\begin{array}{r}
\delta_{E, \min }(H) \leq \min _{1 \leq n<m \leq K_{o}}\left\{\max _{1 \leq k \leq Q_{m}} \delta_{1, k}^{(m)}\left(\underline{\alpha}_{1}^{(m)}\right),\right. \\
\left.\max _{1 \leq k \leq Q_{n}} \delta_{2, k}^{(m, n)}\left(\underline{\alpha}_{2}^{(m, n)}\right)\right\} .
\end{array}
$$

Therefore, following from union bound we have

$$
\begin{aligned}
& \operatorname{Pr}\left\{\delta_{\min }(H) \leq 0\right\} \\
& \leq \sum_{m=1}^{K_{o}}\left(\begin{array}{c}
K_{o} \\
m
\end{array}\right) \operatorname{Pr}\left\{\max _{k} \delta_{1, k}^{(m)}\left(\underline{\alpha}_{1}^{(m)}\right) \leq 0\right\}+ \\
& \sum_{m=1}^{K_{o}}\left(\begin{array}{c}
K_{o} \\
m
\end{array}\right) \sum_{n=1}^{m-1}\left(\begin{array}{c}
m \\
n
\end{array}\right) \operatorname{Pr}\left\{\max _{k} \delta_{2, k}^{(m, n)}\left(\underline{\alpha}_{2}^{(m, n)}\right) \leq 0\right\}
\end{aligned}
$$

Finally the proof is complete after we manage to show

$$
\begin{gathered}
\operatorname{Pr}\left\{\max _{k} \delta_{1, k}^{(m)}\left(\underline{\alpha}_{1}^{(m)}\right) \leq 0\right\} \doteq \mathrm{SNR}^{-d_{m n_{t}, n_{r}}^{*}(m r)}, \\
\operatorname{Pr}\left\{\max _{k} \delta_{2, k}^{(m, n)}\left(\underline{\alpha}_{2}^{(m, n)}\right) \leq 0\right\} \doteq \mathrm{SNR}^{-d_{n n_{t}, n_{r}}^{*}(n r)} . \\
\text { REFERENCES }
\end{gathered}
$$

\section{REFERENCES}

[1] F. Oggier, G. Rekaya, J.-C. Belfiore, and E. Viterbo, "Perfect space time block codes," IEEE Trans. Inf. Theory, vol. 52, no. 9, pp. 3885- 3902, Sept. 2006.

[2] P. Elia, K. R. Kumar, S. A. Pawar, P. V. Kumar, and H.-F. Lu, "Explicit construction of space-time block codes achieving the diversitymultiplexing gain tradeoff," IEEE Trans. Inf. Theory, vol. 52, no. 9, pp. 3869-3884, Sep. 2006.

[3] P. Elia, B. A. Sethuraman, and P. V. Kumar, "Perfect space-time codes for any number of antennas," IEEE Trans. Inf. Theory, vol. 52, no. 11, pp. 3853 - 3868, Nov. 2007.

[4] H. F. Lu, "Explicit constructions of multi-block space-time codes that achieve the diversity-multiplexing tradeoff," IEEE Trans. Inf. Theory, vol. 54, no. 8, pp. 3790-3796, Aug. 2008.

[5] L. Zheng and D. Tse, "Diversity and multiplexing: a fundamental tradeoff in multiple antenna channels," IEEE Trans. Inf. Theory, vol. 49, no. 5, pp. 1073-1096, May 2003.

[6] D. N. C. Tse, P. Viswanath, and L. Zheng, "Diversity-multiplexing tradeoff in multiple-access channels," IEEE Trans. Inf. Theory, vol. 50, no. 9, pp. 1859-1874, Sep. 2004.

[7] M. E. Gärtner and H. Blöcskei, "Multiuser space-time/frequency code design," in Proc. 2006 IEEE Int. Symp. Inform. Theory, Seattle, WA, Jul. 2006, pp. $2819-2823$.

[8] Y. Nam and H. E. Gamal, "On the optimality of lattice coding and decoding in multiple access channels," in Proc. 2007 IEEE Int. Symp. Inform. Theory, Nice, France, Jun. 2007.

[9] Y. Hong and E. Viterbo, "Algebraic multi-user space-time block codes for 2x2 MIMO," in Proc. 2008 IEEE PIMRC, Cannes, France, Sep. 2008.

[10] M. Badr and J.-C. Belfiore, "Distributed space-time block codes for the MIMO multiple access channel," in Proc. 2008 IEEE Int. Symp. Inform. Theory, Toronto, ON, Jul. 2008.

[11] W. Zhang and K. B. Letaief, "A systematic design of multiuser spacefrequency codes for MIMO-OFDM systems," in Proc. 2007 IEEE Int. Conf. Commun., Jul. 2007, pp. 1054-1058. 